\title{
Pflanzliche UV-Filter noch weitgehend unerforscht
}

? Im Bereich der pflanzlichen Wirkstoffe zum Sonnenschutz wird derzeit viel geforscht. Gegenüber synthetischen Filtern verspricht man sich vor allem einen Vorteil in Sachen Allergie. Sind bei pflanzlichen Produkten nicht mindestens ebenso häufig allergische Reaktionen zu erwarten?

Kerscher: Diese Frage kann man sicherlich nicht so allgemein beantworten. In der Tat beobachten wir aber sowohl auf sekundäre Pflanzenstoffe wie auch auf manche synthetische UV-Filter allergische Reaktionen.

? Bei welchen pflanzlichen Sonnenschutzmitteln ist mit einem besonders hohen Allergierisiko zur rechnen?

Kerscher: Ein Sonnenschutzmittel besteht ja nicht nur aus den UV-absorbierenden Substanzen, also den Lichtschutzfiltern, sondern auch aus einer Reihe anderer Inhaltsstoffe, etwa Konservierungsstoffe, Duftstoffe, Cremegrundlagen, um nur einige zu nennen. Eine Allergie vom Typ IV kann auf jeden einzelnen Inhaltsstoff, außer auf Wasser oder Alkohol, entstehen. Zudem ist der Begriff pflanzliches Sonnenschutzmittel per se eher irreführend. Bei den pflanzlichen UV-Filtern handelt es sich typischerweise nicht um UV-Filter im eigentlichen Sinne, sondern meistens um sekundäre Pflanzenstoffe, also beispielsweise Flavonoide oder Vitamine, die eine gewisse UV-Schutzwirkung über antioxidative Effekte bieten, meist allerdings nur im Bereich eines Lichtschutzfaktors fünf bis acht. Möglich sind auch Photosensibilisierungen, also sonnenbrandähnliche Reaktionen durch Pflanzenöle.

? Mit welchen Problemen muss beim Einsatz von pflanzlichem Sonnenschutz außerdem gerechnet werden?

Kerscher: Die Studienlage hierzu ist nicht geeignet, um die Frage valide zu beantworten. Wechselwirkungen zwischen Topika können immer auftreten, vor allem galenische Wechselwirkungen, durch die sich die Produkteigenschaften verändern können. Daher empfiehlt man immer, zwischen der Anwendung mehrerer Topika, die nicht aufeinander abgestimmt sind, 20 bis 30 Minuten Pause.

? Derzeit werden pflanzliche Wirkstoffe eher in Kombination mit synthetischen Filtern genutzt, weil ihr Lichtschutzfaktor allein in der Regel nicht ausreicht. Welche Vorteile haben solche Kombinationen?

Kerscher: Absolut richtig - diese Kombination ist sehr sinnvoll. Auf der einen Seite können synthetische UV-Filter oxidativen Stress nicht vollständig verhindern, auf der anderen Seite bieten Pflanzenstoffe alleine keinen ausreichend hohen UV-Schutz.

? Wenn mit physikalischen Filtern wie Titandioxid oder Zinkoxid aus Nanopartikeln kombiniert wird, kommen da nicht neue Probleme auf? Nanopartikel haben derzeit ja ein relativ schlechtes Image ...

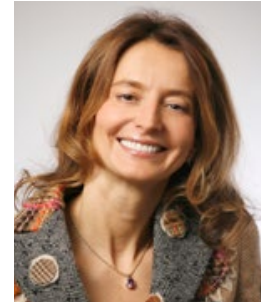

Prof. Dr. med. Martina Kerscher

Universität Hamburg

Abteilung Biochemie und Molekularbiologie

Fachrichtung Kosmetikwissenschaft

Universität Hamburg

Kerscher: Dieses Image ist zum Teil aber auch konstruiert, denn wir setzen etwa Zinkoxid seit Langem in der Dermatologie ein, und zwar ohne Probleme. Die Definition Nanopartikel sagt ja nur etwas über die Größe der Teilchen aus. UV-Licht streuende unbeladene Nanopartikel von Titandioxid oder Zinkoxid mit einer Teilchengröße von $400 \mathrm{~nm}$ etwa dringen nicht in die Haut ein.

? In der Naturkosmetik setzt man auf reine Pflanzenöle. Meist ist der UV-Schutz gering, aber das Karanjaöl (Acorelle) von den Früchten der indischen Buche wird als reines Öl ohne Zusätze mit einem Lichtschutzfaktor von 30 beworben. Was halten Sie davon?

Kerscher: Bislang sind mir dazu keine publizierten wissenschaftlichen Studien bekannt. Jedoch enthalten manche Produkte Karanjaöl mit Titandioxid als physikalischen UV-Filter.

? Sollte der Dermatologe seinen Patienten von der Verwendung von Sonnenölen mit rein pflanzlichen UV-Filtern eher abraten? Kerscher: Aus dermatologischer Sicht raten wir zu einem täglichen UV-Schutz im Bereich LSF 15-30, bei besonderer Belastung, zum Beispiel Outdoor-Aktivitäten, bis LSF 50. Damit ist der rein pflanzliche UV-Schutz durch Pflanzenöle bis dato zu gering.

Das Interview führte Dr. Christine Starostzik. 